Cartas científicas

Rev Urug Cardiol 2021; 36: e

doi:

\title{
Arritmia ventricular maligna asociada a un gossypiboma. Reporte de un caso
}

\author{
Resumen \\ Un gossypiboma, oblitoma o textiloma se define como todo cuerpo extraño olvidado en el interior de un paciente durante \\ una intervención quirúrgica ${ }^{(1)}$. Representa una complicación posquirúrgica poco frecuente ${ }^{(2)}$ pero de consecuencias po- \\ tencialmente graves ${ }^{(3)}$. En general, se manifiesta con cuadros clínicos variables: una masa o pseudotumor, sangrados, \\ fiebre, dolor, varios meses o años después de la cirugía original. También puede ser un hallazgo accidental ${ }^{(4)}$. \\ Describimos el caso clínico de una paciente con un gossypiboma vinculado a una cirugía cardíaca que se realizó 40 años \\ antes al cuadro clínico actual, que se presenta con arritmia ventricular maligna. \\ Palabras clave: GOSSYPIBOMA \\ ARRITMIA VENTRICULAR MALIGNA \\ Malignant ventricular arrhythmia associated to gossypiboma. Clinical case report \\ Summary \\ A gossypiboma, oblitoma or textiloma is defined as a surgical object left in the interior of the body after surgery ${ }^{(1)}$. It re- \\ presents an uncommon ${ }^{(2)}$ but potentially life threatening post-surgery complication ${ }^{(3)}$. Clinical manifestations are varia- \\ ble and depend on location, relations and size, from pseudo-tumoral masses, bleeding, fever, pain and other nonspecific \\ presentations. The onset of symptoms is unpredictable, occurring from months or years after surgery. Patients may \\ course asymptomatically and be diagnosed incidentally as an imaging finding ${ }^{(4)}$. \\ We describe a case of a patient with gossypiboma diagnosed 40 years after undergoing cardiac surgery, who presented \\ with a malignant ventricular arrhythmia. \\ Key words: $\quad$ GOSSYPIBOMA \\ MALIGNANT VENTRICULAR ARRHYTHMIA
}

\section{Arritmia ventricular maligna vinculada a gossypiboma. Reporte de um caso clínico}

\section{Resumo}

Um gossypiboma, oblitoma ou textiloma está definido como todo corpo estranho esquecido no interior de um paciente durante um procedimento cirúrgico ${ }^{(1)}$, representando uma complicação pos cirúrgica pouco frequente ${ }^{(2)}$, mas com consequências potencialmente graves ${ }^{(3)}$. Geralmente, se manifesta com quadros clínicos variáveis: uma massa ou pseudotumor, sangramentos, febre, dor, varios meses ou anos após a cirurgia original ou ser um hachado acidental ${ }^{(4)}$. Descrevemos o caso clínico de uma paciente com um gossypiboma vinculado a uma cirurgia cardíaca realizada 40 anos antes do quadro clínico atual, que se apresenta com arritmia ventricular maligna.

Palavras chave: GOSSYPIBOMA ARRITIMIA VENTRICULAR MALIGNA

\section{Presentación del caso}

Paciente de sexo femenino de 58 años, con antecedentes personales de comunicación interventricular que requirió corrección quirúrgica a los 17 años, hipertensión arterial grado I en tratamiento con amlodipina, flutter auricular con cardioversión eléctrica exitosa en diciembre de 2018. Posteriormente, se mantiene con tratamiento con bisoprolol y anticoagulada con warfarina. Hipotiroidismo en tratamiento con levotiroxina, cirugía bariátrica con realización de manga gástrica en el año 2017.

Enfermedad actual: en agosto de 2019 consulta a unidad de emergencia móvil por palpitaciones, disnea de reposo, dolor precordial acompañado de palidez cutáneo-mucosa y sudoración intensa. Se constata paciente polipneica, con frecuencia cardíaca de 180 cpm y PAS de 60 mmHg. El electrocardiograma de 12 derivaciones realizado (figura 1) muestra una taquicardia regular de complejos anchos a $180 \mathrm{cpm}$, interpretada como taquicardia ventricular monomórfica sostenida. Se procede a cardioversión eléctrica sincronizada, se retoma el ritmo sinusal y después se traslada a emergencia.

$\mathrm{Al}$ ingreso en emergencia la paciente se encontraba clínicamente estable, sin requerimientos de oxígeno ni fármacos, en ritmo sinusal con una frecuencia cardíaca de $50 \mathrm{cpm}$, ruidos cardíacos normofonéticos, sin soplos. No se constatan signos de 


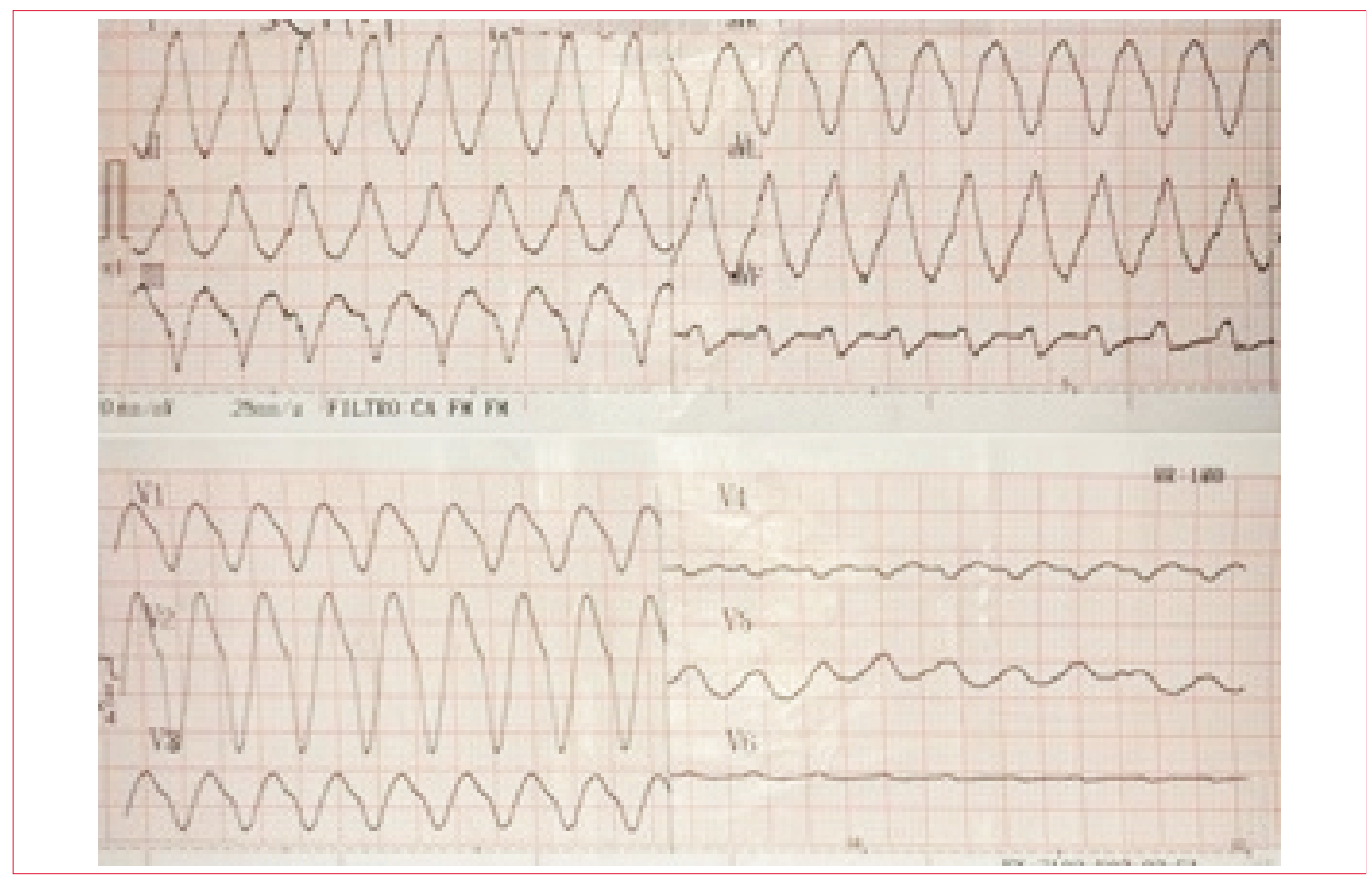

Figura 1. Electrocardiograma de superficie de 12 derivaciones que evidencia taquicardia ventricular monomórfica sostenida a $180 \mathrm{cpm}$, con eje en el plano frontal de $0^{\circ}$ y transición tardía en precordiales.

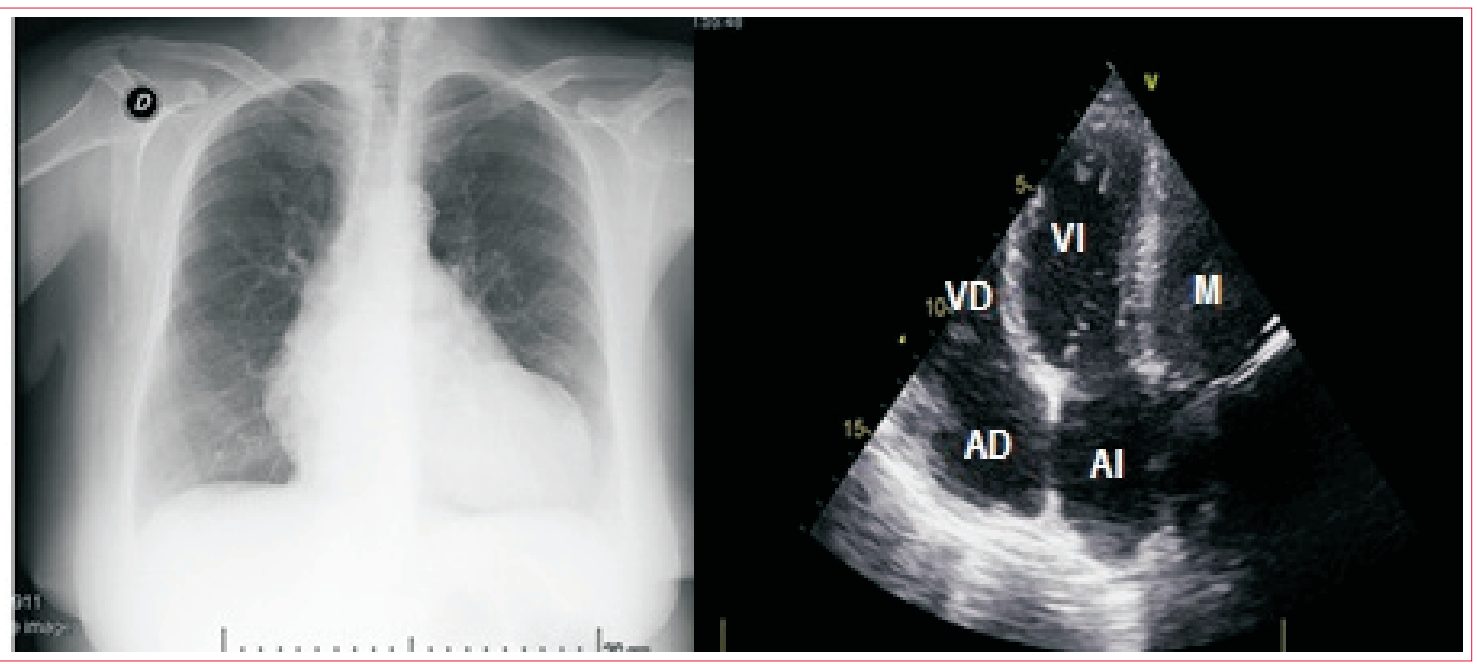

Figura 2. A la izquierda, radiografía de tórax frente en el que se muestra imagen redondeada, delimitada de unos $8 \mathrm{~cm}$ de diámetro con densidad similar a la cardíaca. A la derecha, ecocardiograma transtorácico en ventana apical de cuatro cámaras que visualiza masa extracardíaca en relación con pared lateral del ventrículo izquierdo. AD: aurícula derecha; $\mathrm{AI}$ : aurícula izquierda; VD: ventrículo derecho; VI: ventrículo izquierdo; M: masa paracardíaca.

hipertensión venosa sistémica. Sin estertores crepitantes a la auscultación pulmonar. Los exámenes paraclínicos eran normales.

Se realiza radiografía de tórax de frente (figura 2), que muestra un índice cardiotorácico conservado, sin signos de hipertensión venocapilar pulmonar. Superpuesto a la imagen cardíaca en tercio inferior del hemitórax izquierdo, se observa imagen redondeada, bien delimitada, de $8 \mathrm{~cm}$ de diámetro mayor, con densidad homogénea similar a la de la silueta cardíaca.

El ecocardiograma transtorácico (figura 2) informa una fracción de eyección del ventrículo izquierdo normal y denota una compresión extrínseca de la pared libre del ventrículo izquierdo.

Luego, se realiza tomografía de tórax (figura 3), que describe una imagen compatible con un tumor mediastinal izquierdo en relación con el sector pos- 


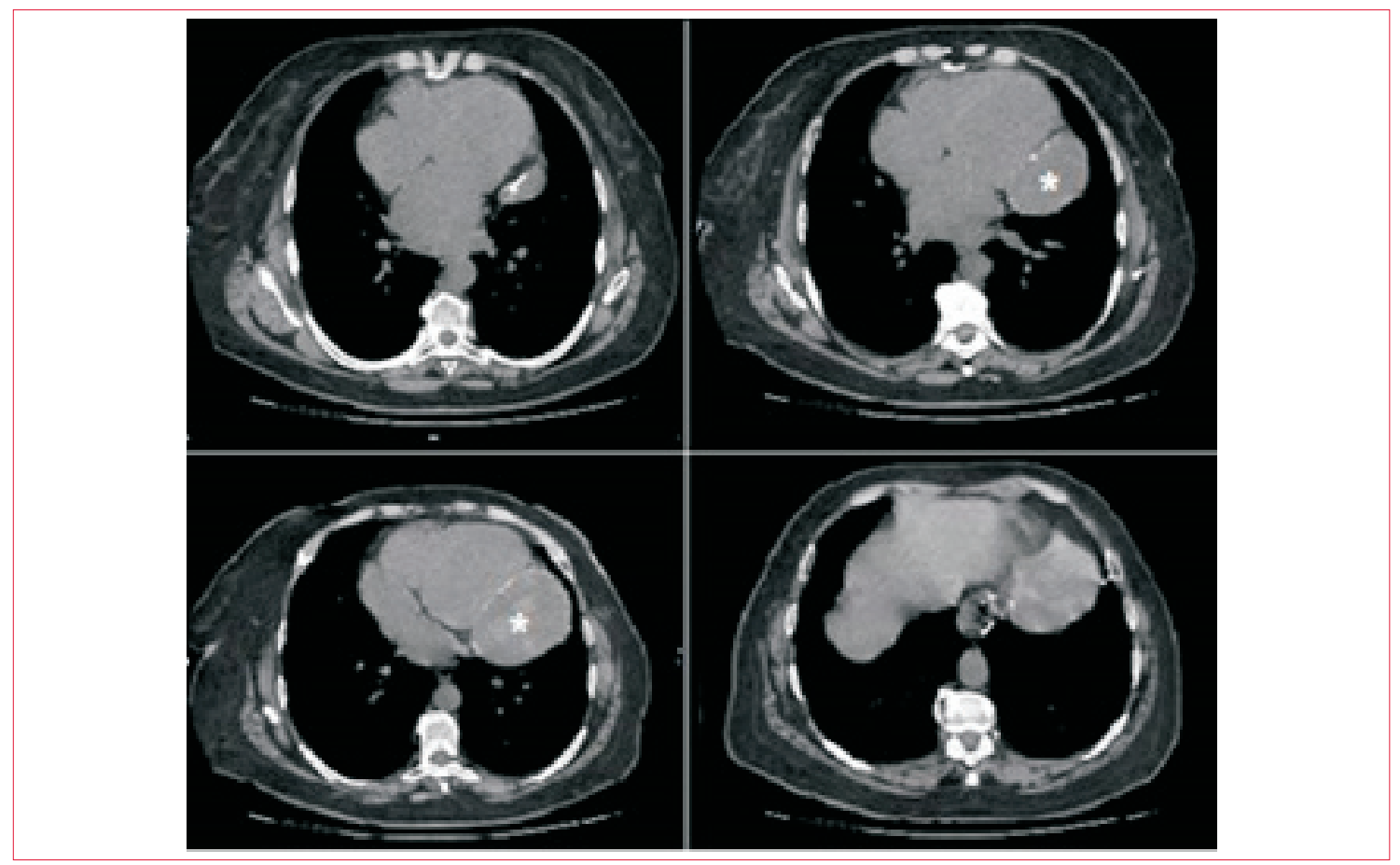

Figura 3. Tomografía computada de tórax ventana mediastinal: se observa en relación posterolateral al ventrículo izquierdo masa sólida heterogénea. * Masa sólida.

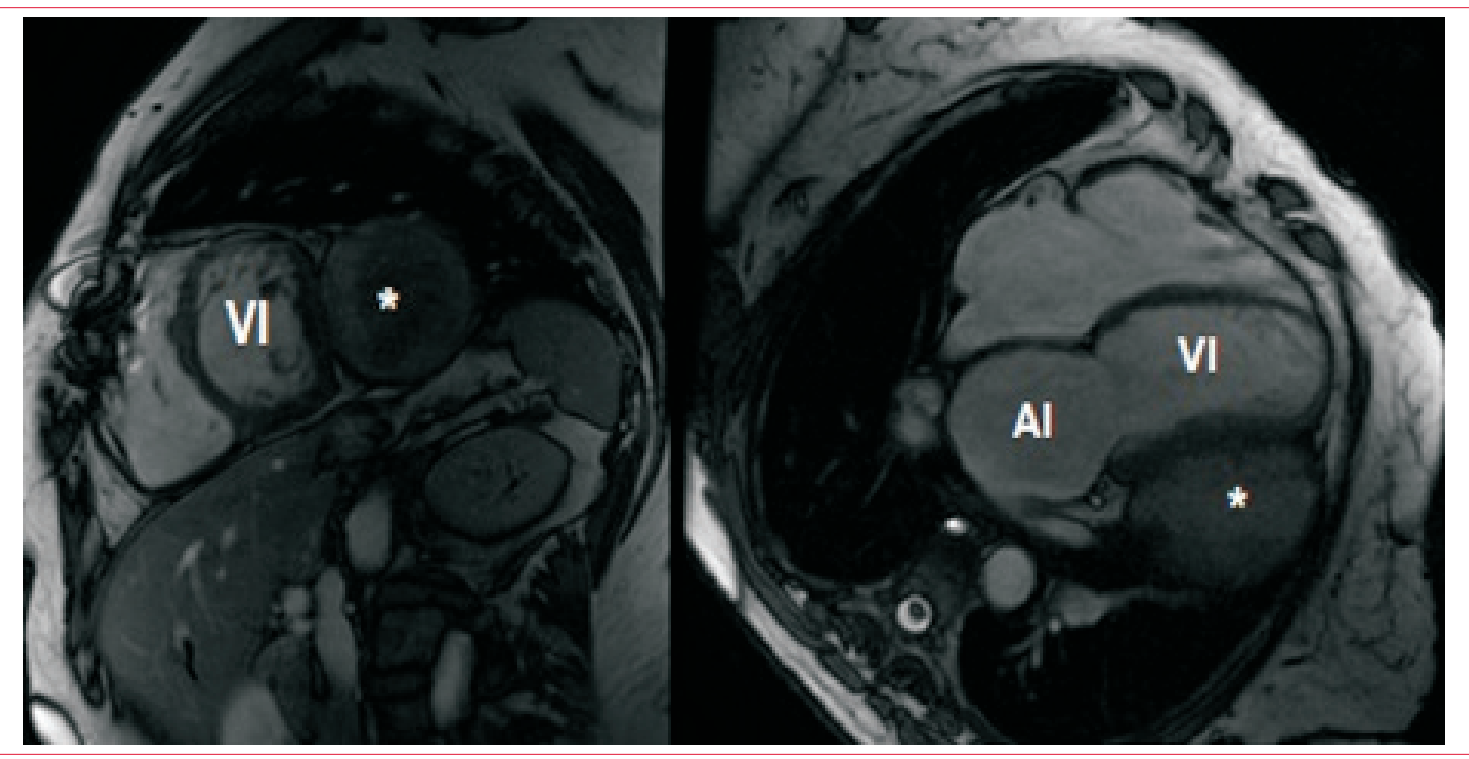

Figura 4. Cardiorresonancia magnética eje corto y cuatro cámaras en la que se evidencia masa que comprime la pared lateral del ventrículo izquierdo. VI: ventrículo izquierdo; AI: aurícula izquierda. * Masa sólida.

terolateral izquierdo de la silueta cardíaca, masa sólida, heterogénea, con áreas hipodensas, de 92 x 79 x $72 \mathrm{~mm}$ en sus diámetros anteroposterior, transversal y longitudinal. En la periferia, en relación con el pericardio parietal, se ven calcificaciones laminares, sin realce con contraste. En el sector retrocardíaco por dentro de la imagen descrita, se ve el tubo gástrico de la cirugía bariátrica referida con las imágenes de alta densidad, características de la sutura mecánica.

Se solicita resonancia magnética cardíaca (figura 4), que muestra compresión extrínseca de la pared lateral del VI por el tumor analizado, bien delimitado, paracardíaco izquierdo, que se encuentra por fuera del pericardio parietal, sin signos de infiltración. En la secuencia de realce tardío a nivel del 


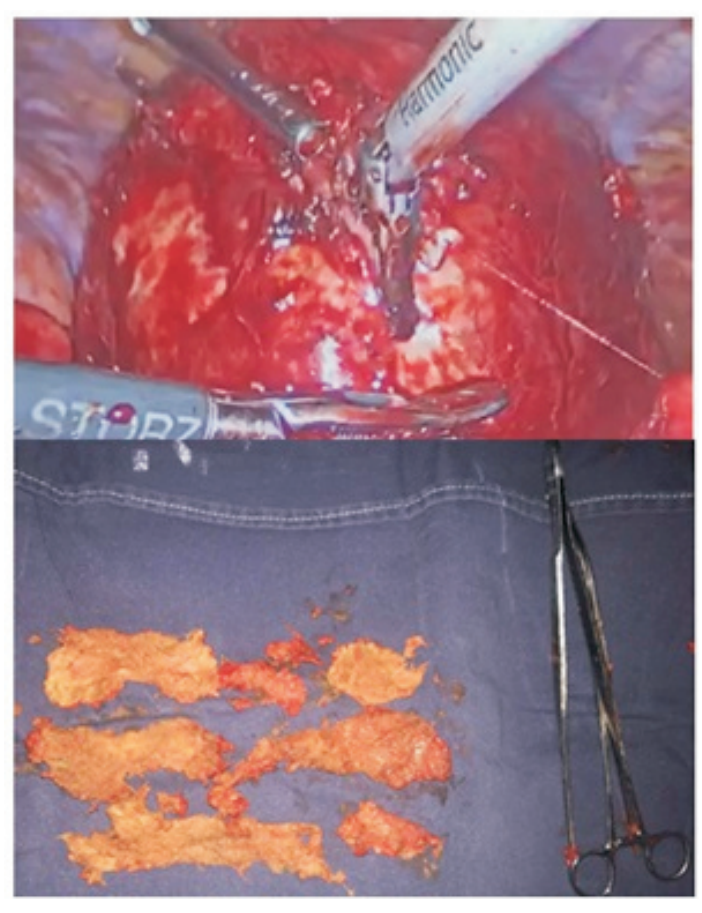

Figura 5. Cardiorresonancia magnética eje corto y cuatro cámaras en la que se evidencia masa que comprime la pa-

septum interventricular en su porción basal y media se observa fibrosis de tipo no isquémica, lineal e intramiocárdica (CIV previa).

Se implanta cardiodesfibrilador como prevención secundaria de muerte súbita. Luego, se realiza videotoracoscopía diagnóstica y terapéutica con resección de cuerpo extraño (figura 5), y se extrae material blanco, que corresponde a una compresa.

La paciente presentó una evolución favorable, sin reiterar episodios arrítmicos ni descargas del CDI.

\section{Discusión}

Un gossypiboma es un pseudotumor que está compuesto de material quirúrgico no absorbible, por lo general es de algodón y corresponde a una gasa o compresa quirúrgica retenida.

$\mathrm{Si}$ bien puede ocurrir en cualquier parte del cuerpo, su localización anatómica intratorácica es excepcional ${ }^{(3)}$. Se han descrito pocos casos en la literatura, la mayoría descubiertos de manera accidental tras realizar diferentes pruebas de imagen por otros motivos clínicos(6).

Su incidencia real es desconocida y se ha señalado que ocurre en 1:100 a 3000 de todos los eventos quirúrgicos; tiene mayor incidencia en cirugías abdominales (1:1000 a 1500 cirugías abdominales)(1).

Varios reportes en la literatura describen las características imagenológicas de los gossypibomas, en especial los de localización intrabdominal; la tomografía computada es el método elegido para su evaluación. El patrón espongiforme con burbujas de gas o la presencia de masas homogéneas de baja densidad con una fina cápsula de alta densidad son los hallazgos más frecuentes ${ }^{(1)}$.

Como se trata de una afección poco común, con serias implicancias médico-legales y manifestaciones clínicas inespecíficas que se pueden acompañar de complicaciones graves, que pueden llegar a ser fatales, es necesario que el clínico, el cirujano y los imagenólogos tengan siempre en mente esta hipótesis diagnóstica y sus formas de presentación, tanto clínicas como imagenológicas, para que un diagnóstico precoz y un tratamiento correcto y definitivo se lleven a cabo.

En el caso presentado, el gossypiboma fue una hipótesis clínica basada en el antecedente de cirugía cardíaca del paciente, junto con los hallazgos de los estudios de imagen realizados. Habitualmente, la mayoría de las masas cardíacas se detecta con ecocardiografía. En este caso, la sospecha inicial fue radiológica. La tomografía computarizada y, sobre todo, la resonancia proporcionan no solo la caracterización del tumor, sino también su localización, tamaño, relaciones, grado de extensión, invasión de tejidos adyacentes y la presencia de derrames aso$\operatorname{ciados}^{(7)}$. Si bien los casos reportados de gossypibomas intratorácicos describen al dolor torácico y la tos como las manifestaciones clínicas más frecuentes ${ }^{(8)}$, el síntoma que motivó la consulta en este caso fueron las palpitaciones. El primer hallazgo fue un ECG que muestra una taquicardia de complejos anchos a $180 \mathrm{cpm}$. No encontramos reportes de casos que asocien gossypibomas paracardíacos con arritmias ventriculares malignas. Planteamos que la descarga neurohumoral generada por la compresión mecánica podría haber actuado como gatillo arritmogénico sobre el sustrato preexistente (fibrosis intramiocárdica por corrección de CIV).

El mejor abordaje de esta situación es su prevención. Se debe utilizar material quirúrgico radiopaco y tomar medidas sencillas esenciales, como vigilar los materiales quirúrgicos, efectuar un conteo meticuloso de estos antes y después del procedimiento, así como la revisión exhaustiva del sitio quirúrgico al final del procedimiento.

\section{Referencias bibliográficas}

1. Motta RGA, González BO, Castillo LJA, Villalobos GE. Material quirúrgico olvidado: gossypiboma, textiloma, gasoma. Anales de Radiología México [Internet]. 2007 (consulta: 7 Jul 2021);6(4): (aprox. 
10p.). Disponible en: https://www.medigraphic.com/ pdfs/anaradmex/arm- 2007/arm074e.pdf

2. Rabie ME, Hosni MH, Al Safty A, Al Jarallah M, Ghaleb FH. Gossypiboma revisited: a never ending issue. Int J Surg Case Rep. 2016;19:87-91. doi: 10.1016/j.ijscr.2015.12.032

3. Arcas JJ, Gracia Martínez J, Cuarental García A, Fajardo Pérez MI. Textiloma intratorácico. A propósito de un caso. Rev Esp Anestesiol Reanim. 2009;56(1):54-6. doi: 10.1016/S0034-9356(09)70325-1

4. Chinelli J, Olivera E, Rodríguez G. Oblitos en cirugía. Análisis de casuística y estrategias para su prevención. Anfamed. 2017;4(2):47-65. doi:10.25184/anfamed $2017 \mathrm{v} 4 \mathrm{n} 2 \mathrm{a} 3$

5. Leal G, Crosta J, Mendoza M, Bastidas M, Ayeranga G, Carboell A, et al. What has been forgotten is forgotten. Gossypibomas. Diagnosis findings. Viena: European Society Radiology; 2018 (consulta: 7 Jul 2021). doi: 10.1594/ecr2018/C-3121. Disponible en: https://epos.myesr.org/poster/esr/ecr2018/C-3121

6. Topal U, Gebitekin C, Tuncel E. Intrathoracic gossypiboma. AJR Am J Roentgenol. 2001;177: 1485-6. doi: 10.2214/ajr.177.6.1771485

7. Bitar P. Computed tomography and magnetic resonance imaging of the heart. Rev Med Clin Condes. 2013;24(1):54-62. doi: 10.1016/S0716-8640(13)70129-9

8. Machado DM, Zanetti G, Araujo Neto CA, Nobre LF, Meirelles GS, Silva JL, et al. Thoracic textilomas: CT findings. J Bras Pneumol. 2014;40(5):535-42. doi:10.1590/s1806-37132014000500010

Dres. Joaquín Vázquez', Rafael Grassi', Andrea Simeone', Siul Salisbury ${ }^{2}$ 1. Servicio de Cardiología del Hospital MacielHospital Maciel. Montevideo, Uruguay. 2. Cirugía de Tórax. Instituto del Tórax. Depto. Cirugía del Hospital de Clínicas. Montevideo, Uruguay.

Joaquín Vázquez, ORCID 0000-0002-9459-9653.

Rafael Grassi, ORCID 0000-0002-3760-5367.

Andrea Simeone, ORCID 0000-0002-8391-091X.

Siul Salisbury, ORCID 0000-0003-4485-9688. 